\title{
Magneto-rotation experiments of micron-sized particles dispersed in a diffused gas medium oriented to reproduce the dust alignment in the interstellar region
}

\author{
C. Uyeda, T. Komatsu, M. Sakakibara, and K. Tanaka \\ Institute of Earth and Space Science, Graduate School of Science, Osaka University, Toyonaka, Osaka 560-0043, Japan \\ Received 19 June 2002 / Accepted 25 October 2002

\begin{abstract}
The magneto-rotations were detected for graphite single-crystal grains dispersed in the diffused gas media for the first time in the pressure range between $300 \mathrm{~Pa}$ to $10^{5} \mathrm{~Pa}$ at a temperature, $T=293 \mathrm{~K}$. Full alignment was achieved at a field intensity of $14 \mathrm{mT}$ for the graphite crystal of $3 \times 10^{-12}$ grms in weight at $300 \mathrm{~Pa}$. The results were consistent with the Langevin theory, in which the balance between field-induced anisotropy energy and the thermal agitation energy were assumed to drive the process. The achieved experimental procedure provides a technical basis to reproduce the magnetic alignment in the diffused and low temperature condition of the star-formation region, considering that the contemporary experiments on magneto-rotation are performed on particles suspended in the liquid media. The experiments at low temperatures below $160 \mathrm{~K}$ can be performed only in the gas medium. The field intensity to achieve magnetic alignment is expected to be considerably small at molecular cloud temperature, due to the decrease of thermal agitation energy and due to the temperature dependence of the paramagnetic anisotropy following the Curie-Weiss law.
\end{abstract}

Key words. ISM: dust, extinction - ISM: general - methods: laboratory

\section{Introduction}

The origin of dust alignment due to the interstellar magnetic field had been investigated for various cosmic regions consisting different gaseous conditions. The cause of dust alignments was discussed mainly in terms of the paramagnetic relaxation induced in the grain particles first proposed by Davis \& Greenstein in 1951. A rotational magnetic field originating in the thermal rotation of the grain was considered to induce the relaxation. The assumption of supra-thermal rotation, in which the ejection of the gas molecules from the grain surface accelerated the rotational velocity of the grains, was widely accepted to be operational in the diffused region (e.g. Pursell 1979; Spitzer \& McGlynn 1979; Lazarian 1995). The efficiency of the above model had been argued for dust alignment in the dense cloud region(e.g. Whittet 1994), since the rotational motion of the dust was considered to be in a thermal equilibrium state with respect to the gas phase.

An alternative mechanism was proposed under the assumption that the field-induced anisotropy energy, originating from the anisotropy of the diamagnetic susceptibility $(\Delta \chi)_{\mathrm{DIA}}$, exceeds the thermal rotational energy which randomizes the direction of the grains. The model was proposed considering that the grains were composed mainly of diamagnetic elements, according to the elemental abundance. The possibility of

Send offprint requests to: C. Uyeda,

e-mail: uyeda@ess.sci.osaka-u.ac.jp relaxation due to the diamagnetic susceptibility has been discussed (Cayrel \& Schatzmann 1954). Some theoretical studies followed this discussion and it was concluded that the effect of diamagnetic relaxation was negligible compared to the paramagnetic relaxation (Greenberg 1969; Purcell 1969). The number of published $(\Delta \chi)_{\text {DIA }}$ data was limited for the inorganic materials at that time (e.g. Gupta 1983), and it was difficult to accept the possibility of magneto-rotation due to diamagnetic anisotropy as a general phenomenon that might have occurred for diamagnetic materials. The $(\Delta \chi)_{\text {DIA }}$ values were obtained recently for a number of basic oxides such as corundum, forsterite, orthoclase, muscovite, scapolite, apophylite, brucite, gibbsite, talc, petalite, ADP, KDP and gypsum due to the results of the $\Delta \chi-T$ measurements on bulk single crystals from room temperature up to $800 \mathrm{~K}$ (Uyeda et al. 2000a; Uyeda et al. 2000b; Uyeda et al. 2000c). It was concluded that the diamagnetic anisotropy originates mainly in the spatial anisotropy of the individual bonding orbital composing the crystal, indicating that the diamagnetic insulators may generally possess a finite amount of diamagnetic anisotropy, with the exception of crystals having cubic symmetry (Uyeda et al. 2000c; Uyeda et al. 2001a).

The above results indicate that the non-magnetic micronsized particles may generally possess a finite amount of efficiency to cause the magneto-rotation due to diamagnetic anisotropy, which could be a new candidate for the cause of magnetic orientation in natural environments. It has also 
become clear according to the $\Delta \chi-T$ measurements mentioned above that the magnetic anisotropy derived from the paramagnetic impurity ions increases considerably with decreasing temperature following the Curie-Weiss Law for various rockforming oxides. The paramagnetic ions, which are the basis of the paramagnetic relaxation model mentioned above, are generally contained in natural materials.

The number of experimental attempts to investigate the problem of cosmic dust alignment is low at present; e.g., Wurm $\&$ Blum (2000) had proposed an alignment due to the gas drift observed in the process of reproducing non-spherical grains. No direct attempts have been made so far to reproduce the static dust-alignment experimentally, due to the difficulty of reproducing the interstellar conditions. The micron-sized grains should be dispersed in a diffused gas medium below $T=100 \mathrm{~K}$ to reproduce the situation. Temperature dependences of the magnetic grain alignment were examined between room temperature and $180 \mathrm{~K}$ by measuring the single-crystal grains of graphite dispersed in liquid ethanol (Chihara et al. 1998). It was confirmed directly for the first time that the magneto-rotation process of grains dispersed in a fluid medium proceeds according to the balance between the Brownian thermal motion of the grain and the field-induced anisotropy energy. The apparatus used in the experiment, however, did not allow measurements below the melting point of ethanol, $T=159 \mathrm{~K}$. The magnetorotation of grain particles dispersed in the gas medium at $1 \mathrm{~atm}$ was realized recently by means of an apparatus newly developed for this purpose (Uyeda et al. 2001b). The method developed serves as an experimental basis to realize the magnetorotation in the diffuse and low temperature conditions of the interstellar regions. The only procedure to realize measurements at interstellar temperatures below $100 \mathrm{~K}$ at present is to disperse the grains in the gas medium and control its temperature.

The effect of the gas pressure of the dispersing medium on the magneto-rotation process is studied in the present work for graphite micro-crystals. It has become clear that measurements of the magneto-rotation process could be performed for grains dispersed in the gas medium having a pressure as low as $300 \mathrm{~Pa}$ in the presence of terrestrial gravity. The significance of investigating the magneto-rotation in the low temperature region is discussed based on the paramagnetic $\Delta \chi$ dependences measured for basic diamagnetic oxides. The physical properties of micron-sized diamagnetic-particles containing paramagnetic impurity moments have not been fully understood in the low temperature region for many of the oxide minerals.

\section{Experimental design}

An apparatus to observe magneto-rotation of grains dispersed in a gas medium of $10^{5} \mathrm{~Pa}$ was designed and assembled (Uyeda et al. 2001b) on the basis of conventional optical methods to detect the magnetic alignment of small particles dispersed in the liquid medium (e.g. Malet \& Dansfeld 1985). A schematic view of the system is described in Fig. 1. The degree of grain alignment is monitored quantitatively by the intensity of a HeNe laser beam transmitted through the sample suspension in a direction parallel to the magnetic field. Graphite is known to

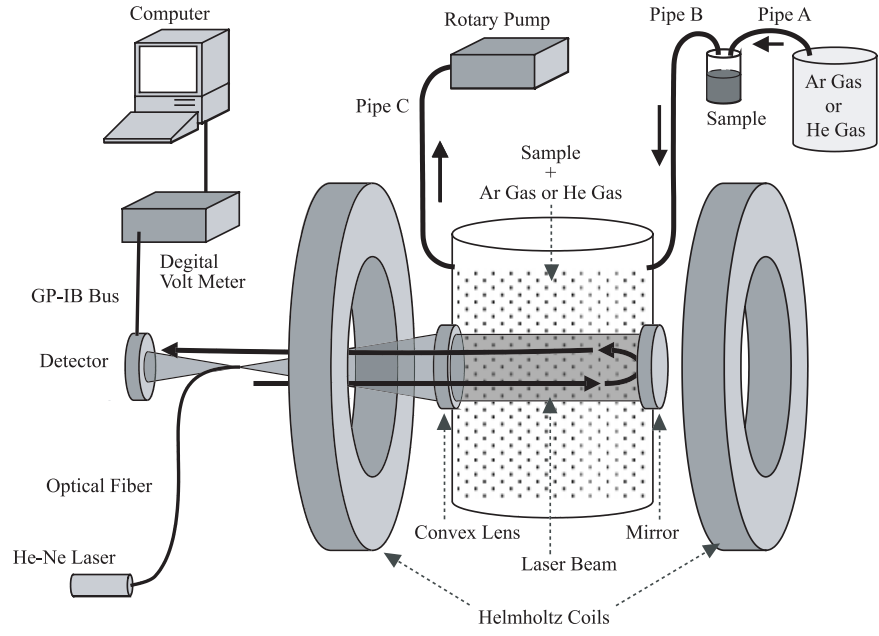

Fig. 1. Schematic view of the experimental setup to measure magneticrotation of micron-sized particles dispersed in the gas phase (Uyeda et al. 2001b).

have an uniaxial type of magnetic anisotropy with its magnetically unstable axis ( $c$-axis) directed perpendicular to the diskplane of the micro-crystal. The disk-planes of graphite are generally developed along the $c$-plane of the crystal. Since the light transmission of graphite is small in the visible region, the intensity of the transmitted light is maximized when the disk-planes are aligned parallel to the direction of the field.

A significant modification had been made to the size of the sample cell with respect to the contemporary system; the inner size of the sample chamber was increased to $200 \mathrm{~mm}$ in diameter and $400 \mathrm{~mm}$ in height. The grains were removed rapidly from the gas phase due to collisions with the inner wall of the cell when the chamber had the conventional size (about $15 \mathrm{~mm}$ in diameter). Quantitative measurements on magnetorotation were prevented by the rapid grain reduction. The transient variation of the transmitted light intensity due to the grain reduction did not disturb the measurements of variations due to the micro-crystal alignments when the chamber size mentioned above was introduced. Consequently the measurement was realized with enough precision to perform quantitative analyses as discussed below.

The chamber was placed at the center of a Helmholtz coil system, which was large enough to produce a homogeneous field (inhomogeneity $<1000 \mathrm{ppm}$ ) up to $75 \mathrm{mT}$ within the spherical area of $500 \mathrm{~mm}$ in diameter at the center of the system. The sample chamber was included inside the spherical area. Consequently, the two equivalent coils had an inner diameter of $1000 \mathrm{~mm}$ and were separated by $570 \mathrm{~mm}$ from each other. Modifications to the optical system were required to detect the degree of alignment, since the density of the grain population per unit volume in the gas medium was significantly lower than that in the liquid suspensions. Quantitative measurements were difficult using the laser beam section (about $\phi 2 \mathrm{~mm}$ in diameter) of the conventional setup since the number of grains intercepting the beam section was low in the gas phase. In the modified system the diameter of the laser beam was increased to $100 \mathrm{~mm}$ by a convex lens and transmitted through the gas suspension as described in Fig. 1. The transmitted light was reflected by a flat mirror back through the 
suspension. The beam was focused finally on the detector by the convex lens. Duplicating the length of the light path in the above manner increased the signal intensity.

Synthetic single-crystal grains of graphite (Columbia Co. Ltd. Japan) were used in the measurement. The magnetic grain alignment had been measured before on the same sample dispersed in the ethanol medium (Chihara et al. 1998), and the $(\Delta \chi)_{\text {DIA }}$ values obtained from the observations were consistent with those of the published data, $(\Delta \chi)_{\text {DIA }}=2.5 \times 10^{-4} \mathrm{emu} / \mathrm{mol}$ (e.g. Gupta 1983). The $\chi-T$ relation of the sample was measured using a vibrating sample magnetometer (Riken Denshi Co., Ltd.,) between $T=300 \mathrm{~K}$ and $77 \mathrm{~K}$. Minor magnetic phases could not be observed above the detection limit of $5 \times 10^{-7} \mathrm{emu} / \mathrm{mole}$. The X-ray diffraction analyses were performed to examine the quality of the crystals and no minor crystal phases were detected according to the analyses.

The grain sample was desiccated at $120^{\circ} \mathrm{C}$ for about $30 \mathrm{hrs}$ and was contained in the ampoule described in Fig. 1, which was connected to the sample chamber by a copper pipe (B). An argon gas tank was connected to the ampoule with a copper pipe (A). The interior of the sample chamber, the ampoule and the copper pipe was exchanged with argon gas supplied from a gas tank. Additional argon gas was then applied from the gas tank into the ampoule with a pressure of $1.1 \mathrm{~atm}$ for about one second, and graphite grains were dispersed into the gas medium. The gas with dispersed graphite was introduced into the sample chamber through pipe (B). The temperature was maintained by an air conditioner of the laboratory at $T=293 \mathrm{~K}$. The pressure of the medium, denoted as $P_{\mathrm{m}}$, was varied from $300 \mathrm{~Pa}$ to $10^{5} \mathrm{~Pa}$ by the use of the vacuum pump system connected to the sample chamber, the pressure being monitored by a Pilany-gage system.

A small portion of the dispersed grains was collected immediately after the experiments by inserting a small copper plate, which was attached at the end of a thin rod, into the center of the chamber interior without altering the value of $P_{\mathrm{m}}$. The rod together with the plate was kept inside a small pipe connected horizontally to the inner space of the chamber during the experiment; the amount of grains attached to the plate during experiment was confirmed to be negligibly small. The graphite grains collected on the copper plate after the experiment were examined visually by an electron probe microanalyzer (JEOL Super Probe 733, Graduate School of Sci., Osaka Univ.). Details of the size analysis were described previously (Chihara et al. 1998). The average areas of the discplanes are listed in Table 1 for the five runs of experiments (S1-S5) measured at different $P_{\mathrm{m}}$ conditions. The thickness of the disc-shaped grains was $0.38 \pm 0.10 \mu \mathrm{m}$ for all the samples according to the above measurements.

The relation was measured between the field intensity $B_{\mathrm{i}}$ and the variation of the transmitted light intensity with respect to the intensity when no magnetic field was applied, denoted as $\Delta I\left(B_{\mathrm{i}}\right)=I\left(B_{\mathrm{i}}\right)-I(0)$. Here, $I\left(B_{\mathrm{i}}\right)$ and $I(0)$ represent the detected light intensity with and without magnetic field $B_{\mathrm{i}}$, respectively. The relation between the degree of grain orientation and the field intensity was calculated from the $\Delta I\left(B_{\mathrm{i}}\right)$ values as described in the next section. The field intensity was increased

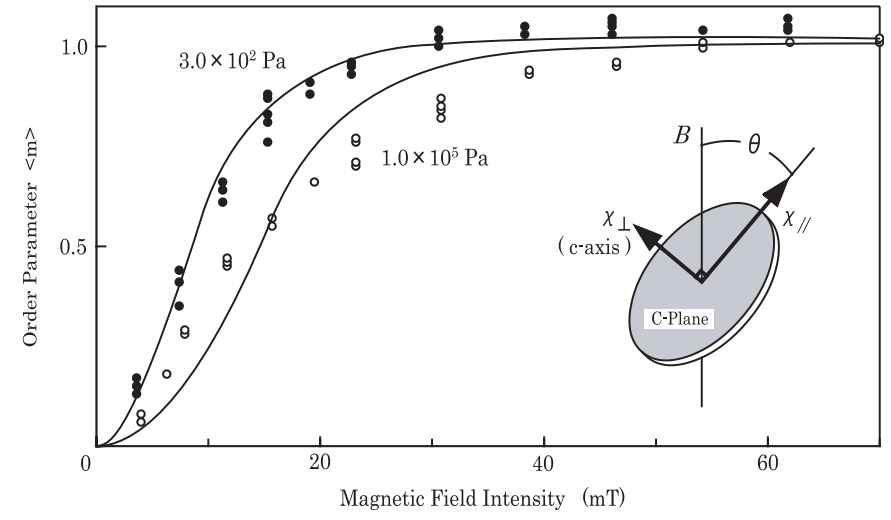

Fig. 2. The relation between the field intensity $B$ and the degree of orientation observed for graphite grains dispersed in the gas medium. Open and solid circles show the observed data measured in the gas medium of $P_{\mathrm{m}}=300 \mathrm{~Pa}$ and $P_{\mathrm{m}}=1.0 \times 10^{5} \mathrm{~Pa}$, respectively. The degree of orientation is described by an order parameter $\langle m\rangle$ defined in the text.

up to the level where $\Delta I\left(B_{\mathrm{i}}\right)$ was saturated at a constant value, $\Delta I\left(B_{\mathrm{S}}\right)$.

The $\Delta I\left(B_{\mathrm{i}}\right)-B_{\mathrm{i}}$ relations were measured for several different $P_{\mathrm{m}}$ conditions as mentioned above. The tangent variation of the light intensity due to turbulence of the gas medium became negligible compared to the $I\left(B_{\mathrm{i}}\right)$ signal when 2-3 min had passed after the introduction of the grain-dispersed gas for the experiment of $\mathrm{S} 1\left(P_{\mathrm{m}}=10^{5} \mathrm{~Pa}\right)$, and the $\Delta I\left(B_{\mathrm{i}}\right)$ signal was detectable for about $1000 \mathrm{~s}$. In the case of $\mathrm{S} 5\left(P_{\mathrm{m}}=300 \mathrm{~Pa}\right)$, argon gas was evacuated slowly after the turbulence had become negligible at $10^{5} \mathrm{~Pa}$. The $\Delta I\left(B_{\mathrm{i}}\right)$ signals could be detected only for $60 \mathrm{~s}$ after the evacuation, indicating that the velocity of grain sedimentation was fast compared to that of S1. The time duration for a set of $\Delta I\left(B_{\mathrm{i}}\right)-B_{\mathrm{i}}$ measurement was less than $30 \mathrm{~s}$ irrespective of $P_{\mathrm{m}}$ values. Quantitative measurements could not be performed below $P_{\mathrm{m}}=300 \mathrm{~Pa}$ since the grains were removed rapidly from the gas phase due to the terrestrial gravity.

\section{Experimental results}

Examples of the relation between the field intensity and the degree of grain-alignment observed for the gas-dispersed graphite crystals are shown in Fig. 2. Solid and open circles represent the observed data measured in the gas medium of $P_{\mathrm{m}}=$ $300 \mathrm{~Pa}(\mathrm{~S} 5)$ and $P_{\mathrm{m}}=1.0 \times 10^{5} \mathrm{~Pa}(\mathrm{~S} 1)$, respectively. The degree of grain-orientation is expressed by an order parameter $\langle m\rangle$, which is commonly used to describe the state of molecular alignment of liquid crystals. The value of $\langle m\rangle$ is defined as the average of the numerical value of a function $\left(3 \cos ^{2} \theta-1\right) / 2$ for the grains orientated in various direction in the suspensions. Here $\theta$ denotes the angle between the directions of the field $B$ and the magnetically stable plane ( $c$-plane) of the disk-shaped crystal, as is described schematically on the right side of Fig. 2. The value of $\langle m\rangle$ is equal to zero when the directions of the grains are in a completely random state, whereas $\langle m\rangle$ is equal to unity when the magnetically stable plane of the grains is aligned completely parallel to the field. 
Table 1. Numerical data of the graphite grains dispersed in argon gas of different pressures. The grain size is described by the area of the disc plane $\left(\mu \mathrm{m}^{2}\right)$ based on SEM observation. The average thickness of the disc-shaped grains was $0.38 \pm 0.1 \mu \mathrm{m}$ for all the samples. The published value of the density of graphite $\rho=2.25\left(\mathrm{~g} / \mathrm{cm}^{3}\right)$ was used to calculate the values of $N .\left(B_{\mathrm{S}}\right)_{\text {theo }}$ and $\left(B_{\mathrm{S}}\right)_{\text {meas }}$ denote the measured and calculated values of the field intensity to achieve magnetic orientation, which is defined by Eq. (2) in the text. The effects of the three parameters $T, N$, and $\Delta \chi$ on the $B_{\mathrm{S}}$ value described in Eq. (2) has been confirmed previously. The experiments to confirm the effect of $T$ has been described in the text (Chihara et al. 1998). The $N$ dependence was confirmed in the range of $N=10^{-10}$ to $10^{-12}$ mole. The $\langle m\rangle-B$ relations were measured for several materials with different $\Delta \chi$ values, namely for kaolin, talc, phlogopite, lepidolite, muscovite and graphite (Uyeda et al. 1991; Uyeda et al. 1993a). The results showed that the $\Delta \chi$ values of individual materials was the dominant factor that determines the $B_{\mathrm{S}}$ value as well.

\begin{tabular}{|c|c|c|c|c|c|c|}
\hline $\begin{array}{l}\text { Sample } \\
\text { No. }\end{array}$ & $\begin{array}{l}P_{\mathrm{m}} \\
(\mathrm{Pa})\end{array}$ & $\begin{array}{l}\text { grain area } \\
\left(\mu \mathrm{m}^{2}\right)\end{array}$ & $\begin{array}{c}N \\
\left(\times 10^{-13} \text { mole }\right)\end{array}$ & $\begin{array}{l}\left(B_{\mathrm{S}}\right)_{\text {meas }} \\
(\mathrm{mT})\end{array}$ & $\begin{array}{l}\left(B_{\mathrm{S}}\right)_{\text {theo }} \\
(\mathrm{mT})\end{array}$ & $\left(B_{\mathrm{S}}\right)_{\text {meas }} /\left(B_{\mathrm{S}}\right)_{\text {theo }}$ \\
\hline S1 & $1.0 \times 10^{5}$ & $2.2 \pm 0.4$ & $1.6 \pm 0.4$ & $22.0 \pm 6.2$ & $12.3 \pm 4.0$ & 1.8 \\
\hline $\mathrm{S} 2$ & $2.0 \times 10^{3}$ & $3.5 \pm 0.4$ & $2.5 \pm 0.6$ & $13.8 \pm 4.0$ & $9.9 \pm 3.0$ & 1.4 \\
\hline S3 & $1.2 \times 10^{3}$ & $4.0 \pm 0.4$ & $2.8 \pm 0.7$ & $11.8 \pm 3.6$ & $9.1 \pm 3.0$ & 1.3 \\
\hline S4 & $7.0 \times 10^{2}$ & $6.0 \pm 0.7$ & $4.3 \pm 1.1$ & $10.8 \pm 3.2$ & $7.5 \pm 2.3$ & 1.4 \\
\hline S5 & $3.0 \times 10^{2}$ & $8.3 \pm 0.7$ & $5.9 \pm 1.5$ & $14.0 \pm 4.6$ & $6.4 \pm 1.6$ & 2.2 \\
\hline
\end{tabular}

The experimental $\langle m\rangle$ values were obtained from the relation $\langle m\rangle=\Delta I\left(B_{\mathrm{i}}\right) / \Delta I\left(B_{\mathrm{S}}\right)$ which was deduced theoretically considering the average amount of light intensity intercepted by the optically-anisotropic grains with their principle-axes distributed in various directions (e.g. Twersky 1969; Maret et al. 1985). The relation was confirmed experimentally for several diamagnetic materials which had published $(\Delta \chi)_{\text {DIA }}$ values (Uyeda et al. 1993; Chihara et al. 1998). The $\Delta I\left(B_{\mathrm{i}}\right) / \Delta I\left(B_{\mathrm{S}}\right)$ values were measured several times for a single field intensity by repeating the $\Delta I\left(B_{\mathrm{i}}\right)$ measurements with and without the magnetic field, following the procedures described in the previous section. It is seen in Fig. 2 that the magnetic alignment at $P_{\mathrm{m}}=300 \mathrm{~Pa}$ is achieved at a relatively low field intensity compared the alignment at $P_{\mathrm{m}}=1.0 \times 10^{5} \mathrm{~Pa}$.

The solid curves of Fig. 2 describe the calculated results fitted for the observed values based on the conventional theory on magnetic alignment of liquid molecules first introduced by Langevin and Curie in 1910. The theory was extended in order to analyze the magneto-rotation of micron-sized particles dispersed in a liquid medium (e.g. Beams 1932; Peterlin \& Stuart 1936; Buckingham \& Polple 1956; Twersky 1969; Maret et al. 1985; Yamagishi \& Date 1989). The thermal agitation of the liquid molecule is assumed to randomize the grain direction. The value of $\langle m\rangle$ is calculated from the Boltzmann average of the function $\left(3 \cos ^{2} \theta-1\right) / 2$ mentioned above at a given temperature $T$. Here the Boltzmann average is calculated in terms of the free energy $U$ induced in a single-crystal grain with the mole number $N$,

$U=-\left(N B^{2} / 2\right)\left\{\chi_{\perp}+\Delta \chi \cos ^{2} \theta\right\}$.

The magnetic anisotropy is defined as $\Delta \chi=\chi_{\|}-\chi_{\perp}$, where $\chi_{\|}$ and $\chi_{\perp}$ denote the molar susceptibility in the direction parallel and perpendicular to the $c$-plane, respectively. It is seen that a $\langle m\rangle-B$ relation is determined by three parameters, namely $T$, $\Delta \chi$ and $N$. The calculated $\langle m\rangle-B$ curves of Fig. 2 gives the best fit to the observed $\langle m\rangle$ values by adopting $N(\Delta \chi)_{\text {DIA }}=$ $(1.3 \pm 0.6) \times 10^{-17} \mathrm{emu}$ for $\mathrm{S} 1\left(P_{\mathrm{m}}=1.0 \times 10^{5} \mathrm{~Pa}\right)$ and adopting $N(\Delta \chi)_{\text {DIA }}=(3.1 \pm 1.4) \times 10^{-17}$ emu for S5 $\left(P_{\mathrm{m}}=300 \mathrm{~Pa}\right)$.

The $\langle m\rangle-B$ relations measured at various $P_{\mathrm{m}}$ values are compiled in Table 1. A parameter had been introduced
(Uyeda et al. 1991; Uyeda et al. 1993) in order to describe quantitatively the field intensity to achieve the magnetic orientation. Complete orientations of the grains (corresponding to $\langle m\rangle=1$ ) could not be obtained at finite field intensity. The field intensity where the order parameter amounts to $\langle m\rangle=0.8$ was therefore defined as $B_{\mathrm{S}}$ which was described as,

$B_{\mathrm{S}}=\left(15 k_{B} T / N \Delta \chi\right)^{1 / 2}$.

It is deduced from the above equations that an arbitrary $\langle m\rangle-B$ relation is determined by a unique $B_{\mathrm{S}}$ value. The observed and calculated $\langle m\rangle-B$ relation could be compared quantitatively by the comparison of the $B_{\mathrm{S}}$ values. The measured $B_{\mathrm{S}}$ value, denoted as $\left(B_{\mathrm{S}}\right)_{\text {meas }}$ in the table, is determined by fitting the theoretical $\langle m\rangle-B$ relation to the observed data as was done in the analysis of Fig. 2. A positive correlation is seen between $P_{\mathrm{m}}$ and $\left(B_{\mathrm{S}}\right)_{\text {meas }}$, as has been seen for the two $\langle m\rangle-B$ curves in Fig. 2. A negative correlation is also evident between $N$ and $\left(B_{\mathrm{S}}\right)_{\text {meas }}$ except for S5 measured at the lowest pressure of $300 \mathrm{~Pa}$. The theoretical $B_{\mathrm{S}}$ values denoted as $\left(B_{\mathrm{S}}\right)_{\text {theo }}$ were obtained by inserting the numerical values in Eq. (2). The following three values were used in the above calculation, namely the published $(\Delta \chi)_{\text {DIA }}$ value of graphite, the room temperature $T=293 \mathrm{~K}$ of the laboratory and the $N$ values obtained from SEM observations mentioned before. The deviations between $\left(B_{\mathrm{S}}\right)_{\text {meas }}$ and $\left(B_{\mathrm{S}}\right)_{\text {theo }}$ values for each measurement are within the mean-square errors except for S5. It may be may be concluded that the reduction of $\left(B_{\mathrm{S}}\right)_{\text {meas }}$ at low $P_{\mathrm{m}}$ values is due to the reduction of the mean size of the measured grains. The grains with relatively smaller sizes may have been preferentially removed from the sample chamber by the evacuation of the argon gas. Consequently the average grain sizes were increased with the reduction of $P_{\mathrm{m}}$, causing an increase of the $\left(B_{\mathrm{S}}\right)_{\text {meas }}$ values. All the $\left(B_{\mathrm{S}}\right)_{\text {meas }}$ values listed in Table 1 were smaller than $22 \mathrm{mT}$, which is exceptionally small for inorganic material. The large $(\Delta \chi)_{\text {DIA }}$ value of graphite, which is more than two orders of magnitude larger than other inorganic materials, is considered to be the cause of the low $B_{\mathrm{S}}$ values.

It is not clear whether the anomalous increase of $\left(B_{\mathrm{S}}\right)_{\text {meas }}$ with respect to $\left(B_{\mathrm{S}}\right)_{\text {theo }}$ observed at the lowest pressure (S5) is due to an intrinsic pressure dependence. The increase of 
gas friction due to the enhancement of the sedimentation velocity in the low pressure condition may increase the fieldinduced anisotropy energy required achieve magneto-rotation. Experiments below $100 \mathrm{~Pa}$, which are difficult to perform with the present apparatus as mentioned before, are required in order to evaluate the cause of this deviation. It is noted that the uncertainties of the $\left(B_{\mathrm{S}}\right)_{\text {meas }}$ values as well as the $\left(B_{\mathrm{S}}\right)_{\text {theo }}$ values are too large to confirm the deviation of S5. The mean square errors amount to $\pm 40 \%$ for the $\left(B_{\mathrm{S}}\right)_{\text {meas }}$ values, which come from the deviation between the measured and theoretical $\langle m\rangle-B$ relations seen in Fig. 2. The deviations are considered to arise from the wide distribution of the grain size contained in a single dispersion sample. In the case of $\left(B_{\mathrm{S}}\right)_{\text {theo }}$ the mean square errors amount to $\pm 30 \%$, which come from the uncertainty of the $N$ values of individual runs of experiments. This shows that the deviation of the $B_{\mathrm{S}}$ value of $\mathrm{S} 1\left(1.0 \times 10^{5} \mathrm{~Pa}\right)$ with respect to the $B_{\mathrm{S}}$ value of the preliminary report (Uyeda et al. 2000a) measured at the same pressure amount to $30 \%$. The difference of the grain-size distribution between different experiments causes the deviation of $B_{\mathrm{S}}$ values between different runs of experiments. Technical improvements are now in progress to reduce the width of the grain-size distribution of the powder samples, since higher precisions are required in the analysis of the $\langle m\rangle-B$ relations.

\section{Discussion}

The magnetic structures in the dense cloud region have been estimated based on polarimetric data (e.g. Tamura et al. 1987; Gerakines et al. 1995), where the field direction was studied with relation to star and planet evolution. The amount of light polarization observed in this region is generally large compared to that of the diffused region. The efficiency of the model based on paramagnetic relaxation is not clear in this region (e.g. Whittet 1994) as mentioned above, where the dust and the gas phase are in a thermal equilibrium state. For example, the dense hot core of Orion KL observed by Rao et al. (1998) was interpreted as the cause of dust alignment, however it was difficult to explain the alignment in terms of the conventional relaxation theory according to the observed conditions. The mechanism of magneto-rotation examined in the present work is effective in the dense cloud condition, since the balance between magnetic-anisotropy energy and the thermal energy provided from the gas molecules controls the process. The experimental results compiled in Table 1 confirm that the mechanism is effective even in the diffuse gas medium of $3 \times 10^{2} \mathrm{~Pa}$, which has a viscosity that is four orders of magnitude smaller than in the conventional experiments performed in liquid media (e.g. Malet \& Dansfeld 1985; Uyeda et al. 1991). These results, along with the recent findings that solid insulators may generally posses a finite $(\Delta \chi)_{\text {DIA }}$ value (Uyeda et al. 2000c; Uyeda et al. 2001a), indicate that the simple magneto-rotation model caused by the magnetic anisotropy of the dust material may be reconsidered as a candidate to explain dust alignment in the dense cloud region. Two conditions should be realized experimentally to judge the efficiency of the model in the molecular cloud condition, namely the temperature and pressure of the gas suspension. Firstly, the temperature should be decreased to $T=10 \mathrm{~K}$. The experiment of magneto-rotation cannot be performed below the melting point of ethanol $(T=158 \mathrm{~K})$ at present. Cryogenic liquids such as $\mathrm{N}_{2}$ or He could not be used as the dispersing medium since micron-sized grains do not disperse efficiently in these liquids. As mentioned before, the unique possibility to realize magnetic grain alignment below $158 \mathrm{~K}$ is to disperse the grain samples in an inert gas, as is done in the present report, and to reduce the temperature of the gas. An apparatus to realize the above experiment can be developed easily by modifying the setup described in Fig. 1, and is now under construction. Secondly, the pressure of the gas phase should be reduced to the order of $10^{-5} \mathrm{~Pa}$, however, the experiments cannot be performed at pressures below $10^{2} \mathrm{~Pa}$ in terrestrial gravity. Experiments are hence being prepared at lower pressure conditions in the micro-gravity facility. The apparatus for this experiment is designed and constructed based on the experimental techniques developed in the present work, since the developed apparatus is effective in detecting the alignment of grains dispersed over a large area and having a low population density.

It is expected from Eq. (2) that the reduction of temperature to the order of $T=10 \mathrm{~K}$ causes the reduction of $B_{\mathrm{S}}$, due to the decrease in thermal rotational energy of the grains and also due to the increase of effective $\Delta \chi$ values derived from the paramagnetic impurity moments. The reduction of $B_{\mathrm{S}}$ caused by the two factors may induce partial dust alignment at the field intensity of the molecular cloud region, which has the order of $100 \mathrm{nT}$. The decrease of the thermal rotational energy at $T=10 \mathrm{~K}$ reduces the $B_{\mathrm{S}}$ value nearly by an order of magnitude with respect to the value observed at room temperature. The $B_{\mathrm{S}}-T$ relation has been examined experimentally only in the range between $T=273 \mathrm{~K}$ and $180 \mathrm{~K}$ as described before (Chihara et al. 1998). It is easily deduced however that the alignment cannot be achieved in molecular cloud field by the reduction of thermal energy alone at $T=10 \mathrm{~K}$.

The effective $\Delta \chi$ values of some sheet silicates were known to increase considerably with decreasing temperature following to the Curie-Weiss Law. The effect was recently observed for various kinds of basic oxide minerals according to the $\Delta \chi-T$ measurements on bulk samples mentioned above performed in the high temperature region (Uyeda et al. 2000c; Uyeda et al. 2001a). The dust alignment due to magnetic anisotropy at a field intensity assumed for the molecular cloud depends on the effective $\Delta \chi$ values of the dust-forming materials at $T=10 \mathrm{~K}$, which can be obtained only through actual $\Delta \chi-T$ measurements of individual materials. Theoretical deduction of the magnetic properties on isolated spins in the diamagnetic crystals is difficult at present. The observation of infrared absorption spectrum in the circumstellar region indicates that the dust particles are partially composed of basic oxide crystals such as corundum $\left[\mathrm{Al}_{2} \mathrm{O}_{3}\right]$ or enstatite $\left[\mathrm{MgSiO}_{3}\right]$. The $\Delta \chi-T$ data at low temperatures should be hence compiled for materials predicted in the dense cloud regions, such as graphite, siliconcarbide, ice and various oxide minerals making the primitive materials.

It is noted however that the $\Delta \chi-T$ measurements on bulk crystals cannot be performed when the size of the crystal is smaller than several millimeters (Uyeda et al. 2000a; 
Uyeda et al. 2001b), which is the case for most of the natural materials. In such cases the $\Delta \chi$ values can be estimated only by analyzing the $\langle m\rangle-B$ curves observed for the micro-crystals, as is done in Fig. 2. The realization of magneto-rotation experiments of grains dispersed in the gas at low temperature conditions are hence essential for determining the actual $\Delta \chi$ values for individual minerals, and for clarifying the possibility of dust alignment due to its magnetic anisotropy in the dense cloud regions.

The experiments in the diffused gas condition are expected to provide numerical data to investigate the mechanism of dust alignment. It was observed that the polarization tended to decrease toward the denser region of the cloud (e.g. Hildebrand et al. 1995; Akeson et al. 1996), which was explained in terms of the growth of the dust aggregates to form a relatively spherical structure or else of the randomization of dust alignment due to the increase in gas-grain collisions. The effect of the thermal energy provided from the gas molecules on the rotational motions of the grains can be examined experimentally by controlling the temperature and pressure of the gas phase as well as the grain sizes.

In conclusion, the experimental procedure achieved in the present work to observe magneto-rotation of gas-dispersed grains is a technical step forward to realize various experimental studies required to solve the mechanism of dust alignment discussed above, taking into account that the contemporary experiments of magneto-rotation on micron sized particles have been performed only in the dense liquid phase fixed at room temperature.

Acknowledgements. The authors wish to thank Dr. J. Blum for his constructive suggestions in improving the manuscript. Thanks are also due to Dr. H. Chihara for helping us with the data analysis. This work was partially supported by the Foundation of Basic Experiments Orientated to Space Station, ISAS, Ministry of Education, Science, Sports and Culture, Japan, by the Grant in Aid for Scientific Research from the Ministry of Education, Science, Sports and Culture, Japan (grant No. 14350008), and also by the Ground Research Foundation of the Japan Space Forum.

\section{References}

Akeson, R. L., Carlstrom, J. E., Phillips, J. A., \& Woody, D. P. 1996, ApJ, 456, L45
Beams, J. W. 1932, Rev. Mod. Phys., 4, 133

Buckingham, A. D., \& Polple, J. A. 1956, Proc. Phys. Soc., 69, 1133

Cayrel, R., \& Schatzmann, E. 1954, Ann. Ap., 17, 555

Chihara, H., Uyeda, C., Tsuchiyama, A., \& Yamanaka, T. 1998, PASJ, 50, 149

Davis, L., \& Greenstein, J. L. 1951, ApJ, 114, 206

Greenberg, J. M. 1969, Physica, 41, 76

Gupta, R. 1983, Landort Bornstein New Ser. II, 445

Hildebrand, R. H., \& Dragovan, M. 1995, ApJ, 450, 663

Malet, G., \& Dransfeld, K. 1985, Topics in App. Phys., 57, 144

Langevin, P., \& Curie, P. 1910, C. R. Acad. Sci. Paris, 15, 331

Lazarian, A. 1995, ApJ, 453, 229

Peterlin, A., \& Stuart, H. A. 1936, Z. Phys., 112, 129

Purcell, E. M. 1969, Physica, 41, 100

Purcell, E. M. 1979, ApJ, 231, 404

Pauling, L. 1936, J. Chem. Phys., 4, 67

Spitzer, L. Jr. 1978, Physical Processes in the Interstellar Medium (Wiley \& Sons Inc., New York)

Spitzer, L. Jr., \& McGlynn, T. A. 1979, ApJ, 231, 417

Twersky, V. 1969, J. Opt. Soc. Am., 60, 1084

Uyeda, C., Takeuchi, T., Yamagishi, A., \& Date, M. 1991, J. Phys. Soc. Jpn., 60, 3234

Uyeda, C. 1993a, Jpn. J. App. Phys., 32, L268

Uyeda, C. 1993b, Phys. Chem. Minerals, 20, 77

Uyeda, C., Takeuchi, T., Yamagishi, A., et al. 1993b, Phys. Chem. Minerals, 20, 369

Uyeda, C., Chihara, H., \& Okita, K. 1998, Phisica B, 246-247, 171

Uyeda, C., Ohtawa, K., \& Okita, K. 2000a, J. Phys. Soc. Jpn., 69, 1019

Uyeda, C., Ohtawa, K., \& Okita, K. 2000b, Jpn. J. App. Phys., 39, L514

Uyeda, C., Ohtawa, K., Okita, K., \& Uyeda, N. 2000c, Jpn. J. App. Phys., 39, L890

Uyeda, C., \& Sakakibara, M. 2001a, J. Phys. Soc. Jpn., 70, 1226

Uyeda, C., Ohtawa, K., Okita, K., \& Uyeda, N. 2001b, J. Phys. Soc. Jpn., 70, 889

Rao, R., Crutcher, R. M., Plambeck, R. L., \& Wright, M. C. 1998, ApJ, 502, L75

Tamura, M., Nagata, T., Sato, S., \& Tanaka, M. 1987, MNRAS, 224, 413

Yamagishi, A., Takeuchi, T., Higashi, H., \& Date, M. 1989, J. Phys. Soc. Jpn., 58, 2280

Whittet, D. C. B. 1992, Dust in the Galactic Environment (Institute of Physics Publishing, Bristol)

Wurm, G., \& Blum, J. 2000, ApJ, 529, L57 